\title{
Article
}

\section{The Immediate effects of sensorimotor foot orthoses on foot kinematics in healthy adults}

Klein, Tomas, Lastovicka, Ondrej, Janura, Miroslav, Svoboda, Zdenek, Chapman, Graham and Richards, James

Available at http://clok.uclan.ac.uk/35567/

Klein, Tomas, Lastovicka, Ondrej, Janura, Miroslav, Svoboda, Zdenek, Chapman, Graham ORCID: 0000-0003-3983-6641 and Richards, James ORCID: 0000-0002-4004-3115 (2021) The Immediate effects of sensorimotor foot orthoses on foot kinematics in healthy adults. Gait and Posture, 84 . pp. 93101. ISSN 0966-6362

It is advisable to refer to the publisher's version if you intend to cite from the work. http://dx.doi.org/10.1016/j.gaitpost.2020.11.022

For more information about UCLan's research in this area go to http://www.uclan.ac.uk/researchgroups/ and search for <name of research Group>.

For information about Research generally at UCLan please go to http://www.uclan.ac.uk/research/

All outputs in CLoK are protected by Intellectual Property Rights law, including Copyright law. Copyright, IPR and Moral Rights for the works on this site are retained by the individual authors and/or other copyright owners. Terms and conditions for use of this material are defined in the policies page.

\section{CLoK}

Central Lancashire online Knowledge www.clok.uclan.ac.uk

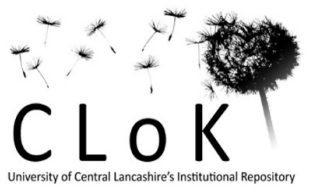


Title:

The Immediate effects of sensorimotor foot orthoses on foot kinematics in healthy adults

\section{Author names and affiliations:}

Tomas Klein ${ }^{1}$ (tomas.klein@upol.cz),

Ondrej Lastovicka1 (ondrej.lastovicka@upol.cz),

Miroslav Janura1 (miroslav.janura@upol.cz),

Zdenek Svoboda1 (zdenek.svoboda@upol.cz),

Graham J Chapman² (GChapman2@uclan.ac.uk),

Jim Richards² (jrichards@uclan.ac.uk).

${ }^{1}$ Faculty of Physical Culture, Palacký University Olomouc, Olomouc, Czech Republic

${ }^{2}$ Allied Health Research Unit, University of Central Lancashire, UK

\section{Corresponding author:}

Tomas Klein; e-mail address: tomas.klein@upol.cz; address: Faculty of Physical Culture, Palacký University Olomouc, třída Míru 117, Olomouc, 77147, Czech Republic

\section{Acknowledgement:}

This work was supported by the Palacký University Olomouc Internal grant [IGA_FTK_2018_014]. 


\section{Highlights:}

Statistical non-parametric mapping explores effect over the entire kinematic curve.

Medial heel bar reduces rearfoot eversion for the majority of the stance phase.

Retrocapital bar rotates rearfoot externally for the entire stance phase. 


\title{
The Immediate effects of sensorimotor foot orthoses on foot kinematics in healthy adults
}

\begin{abstract}
Background: Sensorimotor foot orthoses is an alternative concept, which in addition to mechanical effects, are designed to change muscle activation by altering sensory input to the plantar surface of the foot. However, there is little evidence of how these affect the kinematics of the foot during gait.
\end{abstract}

Research question: The aim of the study was to explore the immediate effect of calcaneal medial heel bars and retrocapital bars on foot kinematics during the stance phase of gait.

Methods: Kinematic data were collected from 32 healthy individuals using an eight camera motion capture system and a six-degrees-of-freedom multi-segment foot model in three different orthotic conditions; calcaneal medial heel bar, retrocapital bar, and no orthosis. Vector field statistical analysis was performed to explore the effect of the orthotic conditions over the kinematic time series curves during stance phase. Peak median and interquartile ranges were also reported during the different phases of stance.

Results: The calcaneal medial bar significantly decreased rearfoot eversion for the majority of the stance phase and compensatory increased midfoot eversion during the entire stance phase compared to the no orthosis condition. The retrocapital bar rotated the foot externally significantly abducting the rearfoot for the entire stance phase and the midfoot for the majority of stance phase.

Significance: The calcaneal medial heel bar and retrocapital bar significantly altered the foot kinematics in a way that may benefit patients with abnormal pronation and intoeing gait. 


\section{Introduction}

Foot orthoses are commonly used to manage various foot related problems [1-5]. Traditional concepts of foot orthoses utilize mechanical effects to adjust the foot biomechanics. However, an alternative concept is the use of sensorimotor elements of insoles which in addition to any mechanical effect, are designed to change muscle activation by altering plantar surface sensory inputs. Sensorimotor insoles comprised of individual components which when combined, can alter lower limb and foot function in Charcot-Marie-Tooth disease [6] and pediatric intoeing gait patients [7]. However, to the authors' knowledge, sensorimotor insoles have not been fully explored to understand the effect of the individual sensorimotor elements on foot kinematics. Recently, Laštovička et al [8] reported a sensorimotor orthosis with a medial heel bar and retrocapital bar with lateral elevation, decreased peak foot eversion, increased peak dorsiflexion and increased peak foot abduction. These findings suggest that the medial heel bar decreases whole foot eversion in a similar fashion to a medial heel wedge which has been reported to reduce rearfoot/ankle eversion [9-13]. Whilst changes to ankle kinematics were reported, this study did not use a multi-segment foot model and as a result was unable to identify subtle foot segment kinematic changes between orthotic conditions [14]. Differences between single-segment and multi-segment foot models have been reported [15], and the importance of midfoot joint motion has been highlighted when describing foot function $[16,17]$. Therefore, the use of a multi-segment foot model may help the understanding of the different components of sensorimotor foot orthoses, such as the effect of the medial heel and retrocapital lateral bars, which may reveal important information when considering their potential clinical use.

The aim of the study was to explore the effect of medial heel and retrocapital bars on foot kinematics during stance phase. We hypothesized that the medial heel bar would decrease rearfoot eversion and increase midfoot eversion between midstance and terminal stance. Whereas, the retrocapital bar would increase midfoot eversion in the second half of stance 
phase and cause external foot rotation by increasing both rearfoot and midfoot abduction throughout stance phase.

\section{Methods}

\section{Participants}

A convenience sample was recruited from members of University staff and students. Participants were included in the study if they had no congenital, or acquired pathology of the nervous or musculoskeletal systems, no deformities or serious injuries of the pelvis or lower limbs and feet, no self-reported lower limb/foot pain, and did not regularly wear foot orthoses. Limb dominance was determined by asking participants which foot they kick a ball with. This study was approved by the Ethical Committee of the Faculty of Physical Culture, Palacký University in Olomouc, Czech Republic (reference number 3/2018) and all participants provided written informed consent prior to data collection.

\section{Procedures}

An eight camera Vicon Vantage V5 motion capture system (Oxford Metrics, UK) was used to collect three-dimensional kinematic data at $200 \mathrm{~Hz}$ within the mid-portion of a $15 \mathrm{~m}$ walkway. Participants were fitted with a pair of correctly sized uniform ProTouch Drop Shot rubbersoled shoes (IIC-INTERSPORT, Bern, Switzerland) and walked, at a self-selected speed, under three different orthotic conditions; no foot orthosis, foot orthoses with a medial heel bar, positioned under the sustentaculum tali, and foot orthoses with a retrocapital bar, positioned posterior to the $2^{\text {nd }}-5^{\text {th }}$ metatarsal heads (Figure 1 ). The medial heel bar and the retrocapital bar were made of polyurethane and had an approximate density of 30 Shore A. The base sole was made of special fabric textile material stiffened with polyethylene. These were part of the NovaPed sensosytem case set (Schein Orthopädie Service KG, Germany). The medial heel bar and the retrocapital bar were attached to the base sole by a trained physiotherapist and differed in scale based on the foot size and no top cover was used. The 
participants received no information about the different configurations of the orthoses used, however they could potentially feel the positions of the bars, therefore these could not be blinded.

Retroreflective markers were placed using the calibrated anatomical system technique (CAST) [18]. Anatomical markers were positioned on the anterior and posterior superior iliac spines, medial and lateral femoral epicondyle, medial and lateral malleoli and over the medial and lateral aspects of first and fifth metatarsal, respectively. Clusters of noncollinear markers were attached to the shank and thigh. Markers were also placed bilaterally over the rearfoot, midfoot and forefoot aspects of the shoes. Anatomical markers were removed after the static trial and tracking markers remained in place during all orthotic conditions and were used to track the segmental kinematics in six degrees of freedom. A single static trial was captured with the participant standing with a comfortable relaxed posture and was used to calculate the ankle and knee joint centres, defined as the midpoints between the lateral and medial malleoli and epicondyles, respectively. Twenty-five walking trials were captured for each condition, the order of which was randomized by participants picking different coloured balls from a bag which represented the different orthotic conditions. A five-minute familiarization period and wash-out time was used between each condition.

\section{Data analysis}

The first stance phase within the mid-portion of the $15 \mathrm{~m}$ walkway from each trial was used for data analysis. Kinematic data were processed in Nexus 2.8 and exported to C3D format. Heel strike and toe-off events were identified manually based on linear acceleration, velocity and visual inspection of the heel and toe marker trajectories. A $4^{\text {th }}$ order low pass Butterworth filter with a $6 \mathrm{~Hz}$ cut-off frequency, and a six-degrees of freedom CAST lower limb model [18] and a multi-segment foot model were applied in Visual 3D (C-Motion, USA) [19]. The X-Y-Z Cardan rotation sequence equivalent to the joint coordinate system was used to calculate joint kinematics [20]. Data were normalized to $100 \%$ of stance phase (heel strike to toe-off) and were averaged for the left and right foot separately. The kinematic variables of interest 
included the peak eversion and abduction values of the rearfoot-to-tibia and midfoot-torearfoot.

Statistical analyses were carried out using spm1d version 0.4 .2 (http://www.spm1d.org/) in Python version 3.7 and in SPSS 25.0 (IBM Corp., USA). A vector field statistical analysis technique, statistical parametric mapping [21] was used to explore the kinematic effects of the orthotic conditions over the whole of stance phase. The D'Agostino-Pearson K2 test was used to assess time series data normality. The non-parametric version of vector field statistical analysis (SnPM) was used [21,22] as not all kinematic data was normally distributed. SnPM One Way ANOVA for repeated measures and SnPM post hoc paired ttests with a Bonferroni correction $(p<0.0167)$ were used to explore for any significant effects between orthotic conditions. For all SnPM tests the number of iterations was set at 10000 . In addition to SnPM analysis, we explored the rearfoot and midfoot peak kinematic values in all three anatomical planes. Shapiro-Wilk tests identified that not all the data were normally distributed, therefore non-parametric Wilcoxon tests were used to explore differences between limbs, and Friedman and Wilcoxon post hoc tests with a Bonferroni correction $(p<0.0167)$ were used to explore the effects between orthotic conditions. Additionally, the effect size ( $r$ ) was calculated [23], with a value of 0.1 to 0.3 being considered a small effect, 0.3 to 0.5 being a moderate effect and 0.5 and above being large effect [24].

\section{Results}

Thirty-two participants (16 males) who were all right side dominant with a mean age, body weight and height of the participants were $22.9 \pm 3.5$ years, $67.9 \pm 10.4 \mathrm{~kg}$ and $173.7 \pm 10.3 \mathrm{~cm}$, respectively. There was no significant difference in walking speed between no orthosis $(1.264 \pm 0.210 \mathrm{~m} / \mathrm{s})$ and retrocapital bar $(1.276 \pm 0.206 \mathrm{~m} / \mathrm{s})$ conditions $(p=0.150)$. Although there was a significant difference in walking speed between no orthosis and medial heel bar $(1.289 \pm 0.207 \mathrm{~m} / \mathrm{s})$ conditions $(p=0.009)$, an increase of $1.98 \%$ was not considered 
meaningful. Wilcoxon tests found no differences between the dominant and non-dominant sides, therefore the differences between orthotic conditions were considered using the data from 64 feet from the 32 participants. Friedman tests showed significant differences in both rearfoot and midfoot eversion and abduction and post hoc Wilcoxon test showed further differences between conditions (Table 1).

The SnPM version of Oneway ANOVA for repeated measures showed significant differences in all three planes for both conditions in rearfoot and midfoot; therefore, only post-hoc findings (Figures 2-5) which were statistically significant for more than $2 \%$ of stance phase were considered meaningful and presented.

\section{Rearfoot}

The medial heel bar significantly increased rearfoot dorsiflexion in the first $5 \%(p<0.001)$ and between $45 \%$ and $85 \%$ of stance phase $(p<0.001)$. Whereas the retrocapital bar significantly decreased dorsiflexion between $50 \%$ and $80 \%$ of stance phase $(p<0.001)$ and increased plantarflexion in the last $5 \%$ of stance phase $(p=0.003)$. In the coronal plane, the medial heel bar significantly decreased eversion for the majority (between $10 \%$ and $80 \%$ ) of stance phase $(p<0.001)$ and peak eversion by $1.29^{\circ} \quad(p<0.001, r=0.5)$. The retrocapital bar significantly decreased eversion in the first $10 \%(p<0.001)$ of stance phase and from $60 \%$ to the end of stance phase $(p<0.001)$. In the transverse plane, the medial heel bar significantly increased rearfoot abduction through the majority of stance phase $(10 \%$ to $90 \%, p<0.001)$ with its peak value increased by $1.25^{\circ}(p<0.001, r=0.4)$, and for the entire stance phase for the retrocapital bar $(p<0.001)$ with its peak value increased by $2.19^{\circ}(p<0.001, r=0.6)$.

\section{Midfoot}

For the midfoot, the medial heel bar significantly increased extension between $5 \%$ and $55 \%$ of stance phase $(p<0.001)$ and decreased extension between $65 \%$ and $80 \%$ of stance phase $(p=0.001)$. The retrocapital bar significantly increased extension for the majority of stance phase (between $20 \%$ and $85 \%, p<0.001$ ). In the coronal plane, the medial heel bar 
significantly increased eversion during the entire stance phase $(p<0.001)$ and peak eversion by $1.31^{\circ}(p<0.001, r=0.6)$. The retrocapital bar significantly increased inversion in the first $15 \%(p<0.001)$ and decreased inversion between $60 \%$ and $75 \%$ of stance phase $(p=0.002)$. In the transverse plane, the medial heel bar significantly increased abduction during the entire stance phase $(p<0.001)$ with its peak value increased by $0.15^{\circ}(p<0.001, r=0.3)$ and the retrocapital bar significantly increased abduction between $20 \%$ and $85 \%$ of stance phase $(p<0.001)$ with its peak value increased by $0.26^{\circ}(p=0.009, r=0.2)$.

\section{Discussion}

This study examined the immediate effect of medial heel and retrocapital sensorimotor bars on foot kinematics. The SnPM explored the effect of the orthotic components across the entire stance phase whilst complementary peak kinematic values were also analysed to highlight the magnitude of the differences between the orthotic conditions. Our findings demonstrated that the two orthotic conditions reduce rearfoot eversion and foot external rotation, respectively. Significant changes were observed in all planes in the rearfoot and midfoot.

The medial heel bar decreased rearfoot eversion for the majority of the stance phase (between 10-80\%) compared to the no orthosis condition, with a median decrease in peak eversion of $1.29^{\circ}$. This could be plausibly explained by an increased inversion moment due to the medial heel bar's shape and position, which is supported by previously reported reductions of the foot eversion when using a medial heel bar [8]. In the midfoot, the medial heel bar increased median peak eversion by $1.31^{\circ}$, which seems to be a compensatory mechanism to decrease eversion in the rearfoot, with the increased eversion in the midfoot lasting the entire stance phase. In previous work, the medial heel bar was reported to cause 'excessive' supination of the foot, which prevented adequate contact of the first metatarsal head and the floor [8]. In contrast, our findings demonstrated reduced rearfoot eversion for 
most of the stance phase and the midfoot appeared to be able to fully compensate for this. However, we also observed the abduction of both rearfoot and midfoot, which would shift the load to the medial structures of the foot [25] and thereby further contributing to the midfoot compensatory eversion.

The medial heel bar increased rearfoot dorsiflexion at heel strike and between $45 \%$ and $85 \%$ of stance phase with its peak at about $75 \%$ of stance phase. This was accompanied by a small increase in flexion of the midfoot between $60 \%$ and $85 \%$ with its peak also at $75 \%$ of stance phase. The increased ankle dorsiflexion was also reported in pediatric intoeing gait patients, where multiple sensorimotor elements, including a medial heel bar, were used together [7]. Both changes in rearfoot and midfoot can be explained by the shape and slightly proximal position of the medial heel bar under the sustentaculum tali, which would possibly increase the dorsiflexion moment of the rearfoot. The increased rearfoot dorsiflexion and midfoot flexion could also be partially caused by altered activity of the tibialis posterior muscle supporting the medial arch of the foot [26] which has been reported to increase in activity in midstance/propulsion phase when under greater load [27]. The coupled motion of increased midfoot eversion, extension and abduction in the first half of the stance phase causing the increased pronation is in accordance with current understanding of the movement of the midtarsal joints [17]. This can be associated with the tendency to flex during terminal stance, especially when emphasized by the mechanical effect of the medial heel bar and potentially altered tibialis posterior activity.

Foot orthoses with medial rearfoot posting have been reported to decrease rearfoot eversion which may benefit patients with abnormal pronation. Past research has reported reductions in peak ankle eversion by $1.1^{\circ}$ and $2^{\circ}$ using either a medial longitudinal arch support with a $5^{\circ}$ rearfoot posting insole [11] or an an anti-pronatory foot orthosis with a $4^{\circ}$ medial heel wedge [12]. Telfer identified a $0.26^{\circ}$ peak rearfoot eversion reduction for every $2^{\circ}$ of posting in the medial direction [13]. In our study, the $25^{\circ}$ medial bar decreased peak eversion by $1.29^{\circ}$, which suggests a minor effect. However, due to the marker placement on the shoe 
and significant differences in material, shape and position between the orthoses, the comparison is rather approximate. The medial heel bar used in this study is made from soft material and is located more distally; therefore, the relatively low immediate effect in the coronal plane rearfoot motion is not surprising. In addition, the purpose of the medial heel bar is not merely a mechanical reduction of eversion, but also a stimulation of the tibialis posterior as a foot invertor in order to achieve long-term change in its activity. Although the particular effect of the medial heel bar on tibialis posterior is beyond this study, future work is needed to determine this and the effect of sensorimotor foot orthoses on muscle activation, as a lateral pressure point is suggested to increase peroneus longus activity during midstance in healthy individuals [28]. On the contrary, there was a minimal effect of sensorimotor insoles on muscle activity in Charcot-Marie-Tooth disease patients. Although, this might have been associated with the neuropathy, reduced proprioception and cavovarus foot deformity rather than the action of the foot orthoses [6].

The retrocapital bar increased midfoot eversion between $60 \%$ and $75 \%$ of stance phase which can be explained by increasing pressure on its position behind the $2^{\text {nd }}-5^{\text {th }}$ metatarsal head. The retrocapital bar decreased rearfoot eversion from about $60 \%$ of stance phase, which as reported previously could be explained as a compensatory mechanism in order to stabilize the foot for propulsion [8]. The retrocapital bar increased midfoot extension for most of stance phase starting from the midstance, which may be explained by an increased extension moment due to its position proximally just behind the $2^{\text {nd }}-5^{\text {th }}$ metatarsal heads. The retrocapital bar also slightly decreased dorsiflexion of the rearfoot between $50 \%$ and $80 \%$ of stance phase which could potentially be explained by either the rearfoot abduction or the differing levels of orthotic material.

The most significant effect of the retrocapital bar was observed in the transverse plane where it increased rearfoot and midfoot abduction and thereby seemed to rotate the entire foot externally, which is consistent with previous findings [8]. The rearfoot was relatively more abducted during the entire stance phase and the increase in peak abduction of $2.19^{\circ}$ 
compared to the no orthosis condition was the largest observed peak increase of all assessed values, which may benefit patients with intoeing gait. The position of the retrocapital bar may have resulted in an increased pressure on the lateral foot structures and therefore plausibly increased the eversion moment. Decrease of the pressure caused by metatarsal pads, which, to some extent, correspond to retrocapital bars, have been reported in previous work $[29,30]$. To decrease the pressure caused by the retrocapital bar, the external rotation of the foot shifted the load to the medial structures of the foot [25]. It is important to note that despite the immediate effects presented here on the sensorimotor orthoses, future research is needed to determine if there are long-term clinical benefits of using such insoles in different patient groups.

This study had limitations. The same static trial was used for all the conditions, which may be a possible source of error. However, to reduce this, all tracking markers remained in place for the entire data collection. This may be the source of between foot and shoe movement error, but all the reported changes were systematic. All participants were young healthy adults therefore the magnitude of any effect may be different for people requiring orthotic interventions and for people of different age groups. A further limitation is that the present study was not blinded. However, blinding with physical devices such as orthoses are extremely difficult given the different appearance of each orthoses.

\section{Conclusion}

This study examined the immediate effect of the medial heel bar and the retrocapital bar sensorimotor elements on the foot kinematics of healthy participants. In addition to the differences in magnitudes of the peak values, this study highlighted the effect of the sensorimotor elements of the components of the foot orthoses over the whole of stance phase using SnPM analysis. These findings support our hypothesis that the medial heel bar significantly decreased eversion of the rearfoot over the majority of stance phase, which may 
benefit patients with rearfoot abnormal pronation. The retrocapital bar significantly increased abduction of both the rearfoot and midfoot for the entire stance phase which may benefit patients with intoeing gait.

\section{Declaration of Interest:}

None

\section{References}

[1] A. Weber, K. Martin, Efficacy of Orthoses for Children With Hypotonia, Pediatr. Phys. Ther. 26 (2014) 38-47. https://doi.org/10.1097/PEP.0000000000000011.

[2] A. Fellas, A. Coda, F. Hawke, Physical and mechanical therapies for lower limb problems in juvenile idiopathic arthritis: a systematic review with meta- analysis., J. Am. Podiatr. Med. Assoc. 107 (2017) 15-213. https://doi.org/10.7547/15-213.

[3] A.H. Vivienne Chuter, Martin Spink, Angela Searle, The effectiveness of shoe insoles for the prevention and treatment of low back pain: a systematic review and metaanalysis of randomised controlled trials, BMC Musculoskelet. Disord. 15 (2014) 140. https://doi.org/10.1177/0269215515570379.

[4] B. Bowring, N. Chockalingam, Conservative treatment of tibialis posterior tendon dysfunction-A review, Foot. 20 (2010) 18-26. https://doi.org/10.1016/j.foot.2009.11.001.

[5] C. McDaid, D. Fayter, A. Booth, J. O'Connor, R. Rodriguez-Lopez, D. McCaughan, R. Bowers, C.P. Iglesias, S. Lalor, R.J. O'Connor, M. Phillips, G. Ramdharry, Systematic review of the evidence on orthotic devices for the management of knee instability related to neuromuscular and central nervous system disorders, BMJ Open. 7 (2017) e015927. https://doi.org/10.1136/bmjopen-2017-015927.

[6] C. Wegener, K. Wegener, R. Smith, K.-H. Schott, J. Burns, Biomechanical effects of sensorimotor orthoses in adults with Charcot-Marie-Tooth disease, Prosthet. Orthot. Int. 40 (2016) 436-446. https://doi.org/10.1177/0309364615579318.

[7] A. Mabuchi, H. Kitoh, M. Inoue, M. Hayashi, N. Ishiguro, N. Suzuki, The Biomechanical Effect of the Sensomotor Insole on a Pediatric Intoeing Gait, ISRN Orthop. 2012 (2012) 1-5. https://doi.org/10.5402/2012/396718.

[8] O. Laštovička, T. Klein, J. Abrantes, M. Janura, Immediate effect of individual bars of insoles and their combination on gait parameters in asymptomatic healthy adults, Somatosens. Mot. Res. 37 (2020) 125-131. https://doi.org/10.1080/08990220.2020.1753686.

[9] C.. Nester, M.. van der Linden, P. Bowker, Effect of foot orthoses on the kinematics and kinetics of normal walking gait, Gait Posture. 17 (2003) 180-187. https://doi.org/10.1016/S0966-6362(02)00065-6. 
[10] C.J. Nester, S. Hutchins, P. Bowker, Effect of Foot Orthoses on Rearfoot Complex Kinematics During Walking Gait, Foot Ankle Int. 22 (2001) 133-139.

https://doi.org/10.1177/107110070102200209.

[11] D. Bonifácio, J. Richards, J. Selfe, S. Curran, R. Trede, Influence and benefits of foot orthoses on kinematics, kinetics and muscle activation during step descent task, Gait Posture. 65 (2018) 106-111. https://doi.org/10.1016/j.gaitpost.2018.07.041.

[12] A. Liu, C.J. Nester, R.K. Jones, P. Lundgren, A. Lundberg, A. Arndt, P. WOLF, Effect of an Antipronation Foot Orthosis on Ankle and Subtalar Kinematics, Med. Sci. Sport. Exerc. 44 (2012) 2384-2391. https://doi.org/10.1249/MSS.0b013e318265df1d.

[13] S. Telfer, M. Abbott, M.P.M. Steultjens, J. Woodburn, Dose-response effects of customised foot orthoses on lower limb kinematics and kinetics in pronated foot type, J. Biomech. 46 (2013) 1489-1495. https://doi.org/10.1016/j.jbiomech.2013.03.036.

[14] A. Leardini, M.G. Benedetti, L. Berti, D. Bettinelli, R. Nativo, S. Giannini, Rear-foot, mid-foot and fore-foot motion during the stance phase of gait, Gait Posture. 25 (2007) 453-462. https://doi.org/10.1016/j.gaitpost.2006.05.017.

[15] C. Pothrat, G. Authier, E. Viehweger, E. Berton, G. Rao, One- and multi-segment foot models lead to opposite results on ankle joint kinematics during gait: Implications for clinical assessment, Clin. Biomech. 30 (2015) 493-499.

https://doi.org/10.1016/j.clinbiomech.2015.03.004.

[16] A.E. Hunt, R. M. Smith, M. Torode, A.-M. Keenan, Inter-segment foot motion and ground reaction forces over the stance phase of walking, Clin. Biomech. 16 (2001) 592-600. https://doi.org/10.1016/S0268-0033(01)00040-7.

[17] C.-B. Phan, G. Shin, K.M. Lee, S. Koo, Skeletal kinematics of the midtarsal joint during walking: Midtarsal joint locking revisited, J. Biomech. 95 (2019) 109287. https://doi.org/10.1016/j.jbiomech.2019.07.031.

[18] A. Cappozzo, F. Catani, U. Della Croce, A. Leardini, Position and orientation in space of bones during movement: anatomical frame definition and determination, Clin. Biomech. 10 (1995) 171-178. https://doi.org/10.1016/0268-0033(95)91394-T.

[19] F. Mager, J. Richards, M. Hennies, E. Dötzel, A. Chohan, A. Mbuli, F. Capanni, Determination of Ankle and Metatarsophalangeal Stiffness During Walking and Jogging, J. Appl. Biomech. 34 (2018) 448-453. https://doi.org/10.1123/jab.2017-0265.

[20] E.S. Grood, W.J. Suntay, A Joint Coordinate System for the Clinical Description of Three-Dimensional Motions: Application to the Knee, J. Biomech. Eng. 105 (1983) 136-144. https://doi.org/10.1115/1.3138397.

[21] T.C. Pataky, J. Vanrenterghem, M.A. Robinson, Zero- vs. one-dimensional, parametric vs. non-parametric, and confidence interval vs. hypothesis testing procedures in onedimensional biomechanical trajectory analysis, J. Biomech. 48 (2015) 1277-1285. https://doi.org/10.1016/j.jbiomech.2015.02.051.

[22] T. Nichols, A. Holmes, Nonparametric Permutation Tests for Functional Neuroimaging, in: Hum. Brain Funct., Elsevier, 2004: pp. 887-910. https://doi.org/10.1016/B978012264841-0/50048-2.

[23] C.O. Fritz, P.E. Morris, J.J. Richler, Effect size estimates: Current use, calculations, and interpretation., J. Exp. Psychol. Gen. 141 (2012) 2-18. https://doi.org/10.1037/a0024338.

[24] J. Cohen, The Significance of a Product Moment rs, in: Stat. Power Anal. Behav. Sci., 2nd ed., Elsevier, London, 1977: pp. 75-107. https://doi.org/10.1016/B978-0-12- 
179060-8.50008-6.

[25] D. Rosenbaum, Foot loading patterns can be changed by deliberately walking with intoeing or out-toeing gait modifications, Gait Posture. 38 (2013) 1067-1069.

https://doi.org/10.1016/j.gaitpost.2013.04.001.

[26] T. Kamiya, E. Uchiyama, K. Watanabe, D. Suzuki, M. Fujimiya, T. Yamashita, Dynamic effect of the tibialis posterior muscle on the arch of the foot during cyclic axial loading, Clin. Biomech. 27 (2012) 962-966.

https://doi.org/10.1016/j.clinbiomech.2012.06.006.

[27] G.S. Murley, H.B. Menz, K.B. Landorf, Foot posture influences the electromyographic activity of selected lower limb muscles during gait, J. Foot Ankle Res. 2 (2009) 35. https://doi.org/10.1186/1757-1146-2-35.

[28] O. Ludwig, J. Kelm, M. Fröhlich, The influence of insoles with a peroneal pressure point on the electromyographic activity of tibialis anterior and peroneus longus during gait, J. Foot Ankle Res. 9 (2016) 33. https://doi.org/10.1186/s13047-016-0162-5.

[29] A.-H. Chang, Z.U. Abu-Faraj, G.F. Harris, J. Nery, M.J. Shereff, Multistep Measurement of Plantar Pressure Alterations Using Metatarsal Pads, Foot Ankle Int. 15 (1994) 654-660. https://doi.org/10.1177/107110079401501205.

[30] L. Jackson, J. Binning, J. Potter, Plantar Pressures in Rheumatoid Arthritis Using Prefabricated Metatarsal Padding, J. Am. Podiatr. Med. Assoc. 94 (2004) 239-245. https://doi.org/10.7547/0940239.

Author statement

Tomas Klein: Conceptualization, Methodology, Investigation, Data Curation, Formal Analysis, Visualization, Writing - Original draft preparation. Ondrej Lastovicka: Conceptualization, Methodology, Investigation. Miroslav Janura: Conceptualization, Writing - Review \& Editing. Zdenek Svoboda: Methodology, Writing - Review \& Editing. Graham J Chapman: Formal Analysis, Writing Review \& Editing. Jim Richards: Conceptualization, Methodology, Formal Analysis, Writing - Review \& Editing. 


\section{Figure Captions}

Figure 1. Diagram illustrating the dorsal (top), lateral (middle) and medial (bottom) aspects of the two sensorimotor insoles; medial heel bar (left) and retrocapital bar with lateral elevation (right).

Figure 2. Diagram illustrating the mean rearfoot kinematic curves for no orthosis (solid line) and medial heel bar (dashed line) condition in sagittal, coronal and transverse plane (left column) with corresponding post hoc SnPM paired t-test results (right column). Positive angles indicate dorsiflexion in sagittal plane, eversion in coronal plane, abduction in transverse plane.

Figure 3. Diagram illustrating the mean midfoot kinematic curves for no orthosis (solid line) and medial heel bar (dashed line) condition in sagittal, coronal and transverse plane (left column) with corresponding post hoc SnPM paired t-test results (right column). Positive angles indicate extension in sagittal plane, eversion in coronal plane, abduction in transverse plane.

Figure 4. Diagram illustrating the mean rearfoot kinematic curves for no orthosis (solid line) and retrocapital bar (dashed line) condition in sagittal, coronal and transverse plane (left column) with corresponding post hoc SnPM paired t-test results (right column). Positive angles indicate dorsiflexion in sagittal plane, eversion in coronal plane, abduction in transverse plane.

Figure 5. Diagram illustrating the mean midfoot kinematic curves for no orthosis (solid line) and retrocapital bar (dashed line) condition in sagittal, coronal and transverse plane (left column) with corresponding post hoc SnPM paired t-test results (right column). Positive angles indicate extension in sagittal plane, eversion in coronal plane, abduction in transverse plane. 
Table 1

Friedman ANOVA comparing kinematic median (25th; 75th Percentil) values during the stance phase under the different orthotic cc

\begin{tabular}{|c|c|c|c|c|c|}
\hline & no orthosis & medial heel bar & retrocapital bar & \multicolumn{2}{|c|}{$p$-value } \\
\hline \multicolumn{6}{|c|}{ rearfoot (degrees) } \\
\hline peak eversion & $\begin{array}{c}10.52 \\
(8.44 ; 13.07)\end{array}$ & $\begin{array}{c}9.29 \\
(6.81 ; 12.23)\end{array}$ & $\begin{array}{c}10.94 \\
(8.38 ; 13.30)\end{array}$ & \multicolumn{2}{|c|}{$<0.001$} \\
\hline peak abduction & $\begin{array}{c}7.51 \\
(5.59 ; 10.28)\end{array}$ & $\begin{array}{c}8.76 \\
(6.54 ; 11.02)\end{array}$ & $\begin{array}{c}9.7 \\
(7.79 ; 12.49)\end{array}$ & \multicolumn{2}{|c|}{$<0.001$} \\
\hline \multicolumn{6}{|c|}{ midfoot (degrees) } \\
\hline peak eversion & $\begin{array}{c}-5.19 \\
(-7.79 ;-3.32)\end{array}$ & $\begin{array}{c}-3.88 \\
(-6.85 ;-1.76)\end{array}$ & $\begin{array}{c}-4.95 \\
(-7.48 ;-3.16)\end{array}$ & \multicolumn{2}{|c|}{$<0.001$} \\
\hline peak abduction & $\begin{array}{c}-0.55 \\
(-2.27 ; 1.13)\end{array}$ & $\begin{array}{c}-0.41 \\
(-2.32 ; 1.42)\end{array}$ & $\begin{array}{c}-0.3 \\
(-2.20 ; 1.24)\end{array}$ & \multicolumn{2}{|c|}{0.001} \\
\hline \multicolumn{6}{|c|}{ Statistically significant pairwise comparisons between the orthotic conditions during the stance phase } \\
\hline & \multicolumn{2}{|c|}{ Comparison between conditions } & $\begin{array}{c}\text { median diff. } \\
\text { (degrees) }\end{array}$ & $p$-value & $r$ \\
\hline \multicolumn{6}{|c|}{ rearfoot (degrees) } \\
\hline \multirow[t]{2}{*}{ peak eversion } & no orthosis & medial heel bar & 1.29 & $<0.001$ & 0.5 \\
\hline & medial heel bar & retrocapital bar & -1.65 & $<0.001$ & 0.5 \\
\hline \multirow[t]{3}{*}{ peak abduction } & no orthosis & medial heel bar & -1.25 & $<0.001$ & 0.4 \\
\hline & no orthosis & retrocapital bar & -2.19 & $<0.001$ & 0.6 \\
\hline & medial heel bar & retrocapital bar & -0.94 & $<0.001$ & 0.4 \\
\hline \multicolumn{6}{|c|}{ midfoot (degrees) } \\
\hline \multirow[t]{2}{*}{ peak eversion } & no orthosis & medial heel bar & -1.31 & $<0.001$ & 0.6 \\
\hline & medial heel bar & retrocapital bar & 1.08 & $<0.001$ & 0.6 \\
\hline \multirow[t]{2}{*}{ peak abduction } & no orthosis & medial heel bar & -0.15 & $<0.001$ & 0.3 \\
\hline & no orthosis & retrocapital bar & -0.26 & 0.009 & 0.2 \\
\hline
\end{tabular}

Bonferroni correction: 


$$
\underline{2}
$$


Figure 2.
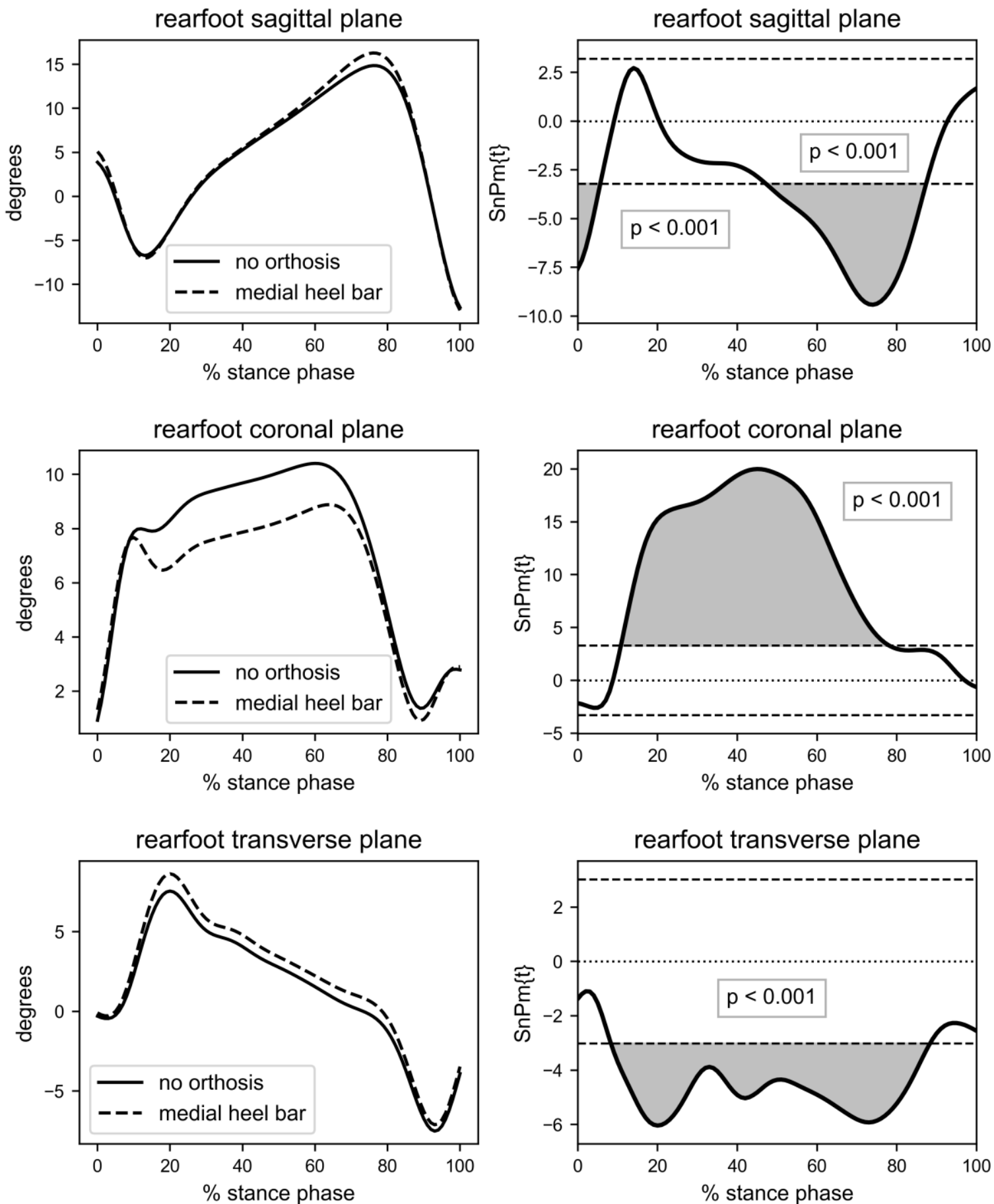

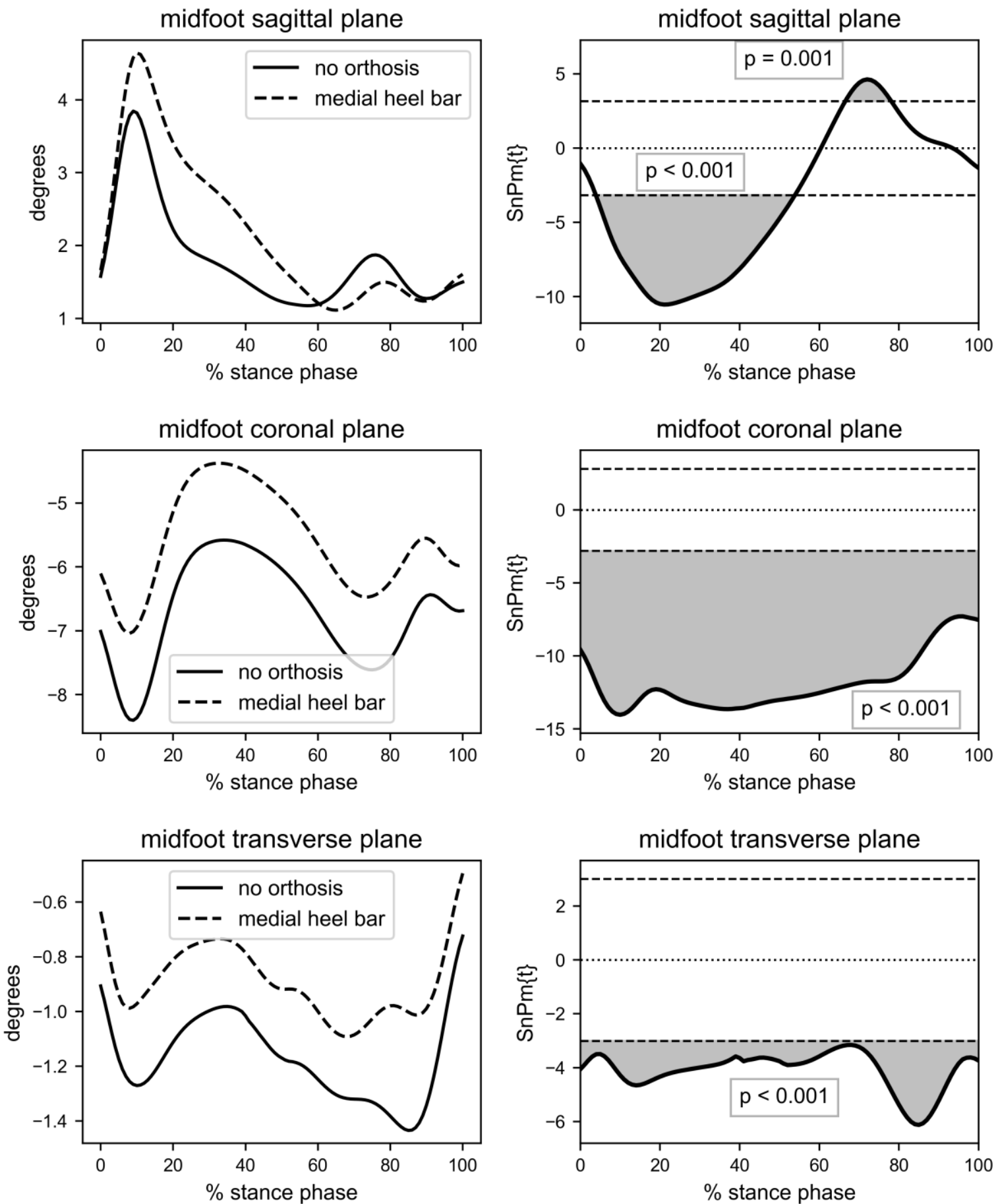
Figure 4.
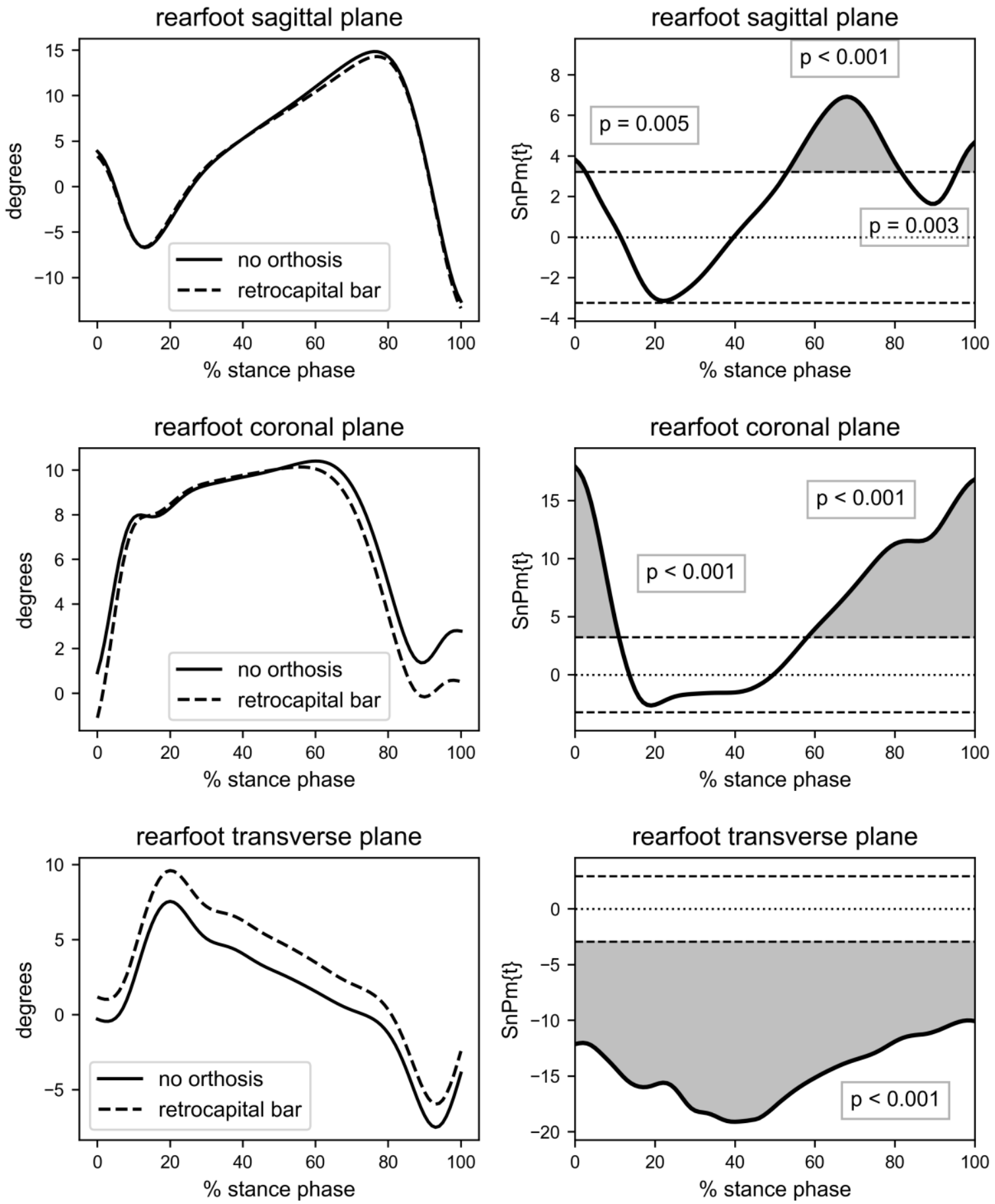
Figure 5.
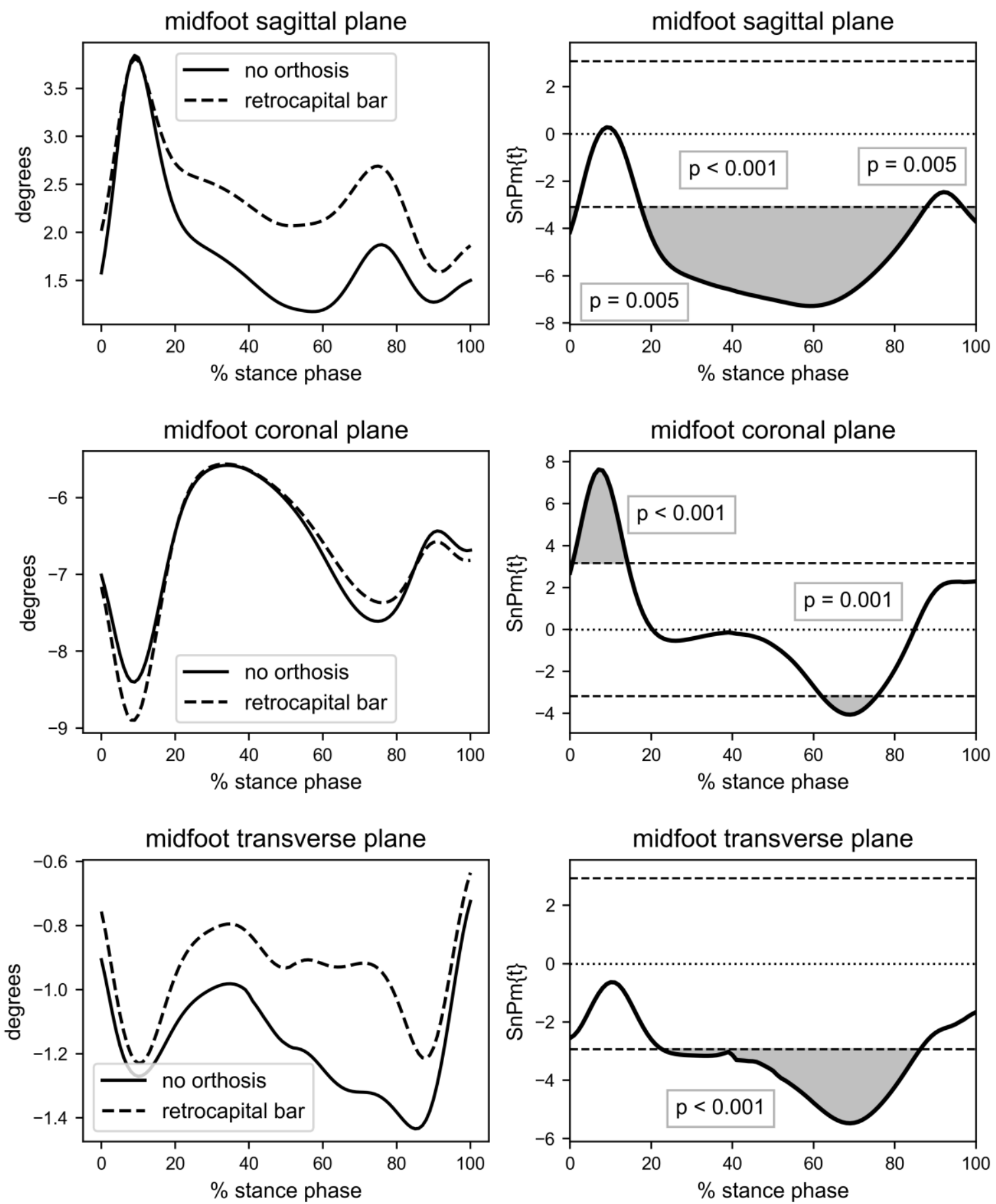